\title{
Spontaneous epidural hematoma secondary to bone infarction in sickle cell anemia: case report
}

\author{
Rohini Komarla, MD, ${ }^{1}$ Bruno P. Soares, MD, ${ }^{2}$ Joshua J. Chern, MD, PhD, ${ }^{3,4}$ and Sarah S. Milla, MD1,4 \\ Departments of ${ }^{1}$ Radiology and ${ }^{3}$ Neurosurgery, Emory University School of Medicine; ${ }^{4}$ Children's Healthcare of Atlanta-Egleston \\ Children's Hospital, Atlanta, Georgia; and 2Division of Pediatric Radiology and Pediatric Neuroradiology, The Johns Hopkins \\ University School of Medicine, Baltimore, Maryland
}

\begin{abstract}
Spontaneous epidural hematoma $(E D H)$ is a rare occurrence in patients with sickle cell disease, with a small number of cases reported. Appropriate diagnosis is critical, because rapid neurosurgical intervention may be required. This unique case illustrates clinical and MRI features of an 18-year-old woman presenting with a headache and subsequent progression to severe focal neurological symptoms. Imaging demonstrated a large EDH of mixed signal characteristics and underlying calvarial infarction, requiring emergency decompression and evacuation. A second companion case is also presented. The authors discuss proposed pathophysiology of the formation of EDHs in sickle cell anemia.

https://thejns.org/doi/abs/10.3171/2018.1.PEDS17407
\end{abstract}

KEYWORDS epidural hematoma; sickle cell anemia; bone infarction; trauma

$\mathrm{N}$ EUROLOGICAL manifestations of sickle cell disease typically include silent and acute parenchymal infarctions as well as moyamoya vasculopathy. Presenting neurological symptoms may include focal deficits, such as limb weakness or slurring of speech, or be nonspecific, such as headaches. Whereas the incidence of headaches in children with sickle cell anemia is relatively close to that in healthy children, there is a higher prevalence of acute cerebrovascular pathology in children with sickle cell disease (6.9\%) compared to healthy children $(0.5 \%){ }^{4}$ Spontaneous epidural hemorrhage is a rare complication of sickle cell anemia and can present with acute headache and neurological symptoms, as in this illustrative case.

\section{Case Report}

History and Examination

An 18-year-old African American woman with a history of sickle cell anemia presented to our pediatric emergency department with chest pain radiating to her lower back, typical of her past sickle cell crises. She also reported a 4-hour history of headache described as throbbing, most severe at the vertex, associated with photophobia, and progressively worsening despite pain medications. Headaches were not a feature of her past pain crises. She denied any history of head trauma. She was admitted for an acute pain crisis and was started on intravenous fluids and morphine.

\section{Neuroimaging}

On the day after hospital admission, the patient reported worsening headache now radiating down the side of her face, and dizziness upon standing. On reassessment she was difficult to arouse, with slurred speech and left arm weakness. Symptoms did not resolve with naloxone. Emergency MRI and MRA of the brain obtained without contrast were performed due to concern for an acute stroke. The MRI revealed a large right-hemispheric epidural hematoma (EDH) measuring $3.1 \mathrm{~cm}$ in thickness, containing blood products of mixed signal characteristics with a $1-\mathrm{cm}$ midline shift (Figs. 1 and 2). There was otherwise no acute parenchymal abnormality. The MRI also revealed heterogeneous signal intensity within the overlying parietal bone on T2-weighted images, consistent with a calvarial bone infarction. Results of the MRA were normal. Of note, the patient had been admitted 7 months earlier for a possible generalized seizure or postsyncopal seizure, and had a normal head CT and MRI findings at the time (Fig. 3). The patient's history of sickle cell anemia was the only known risk factor for bone infarction and bleeding. Additional hematological workup was not performed.

\section{Operation and Postoperative Course}

On neurosurgical evaluation following MRI, the patient was not responding to questions appropriately and had right eye deviation with a sluggish pupillary reflex. She 


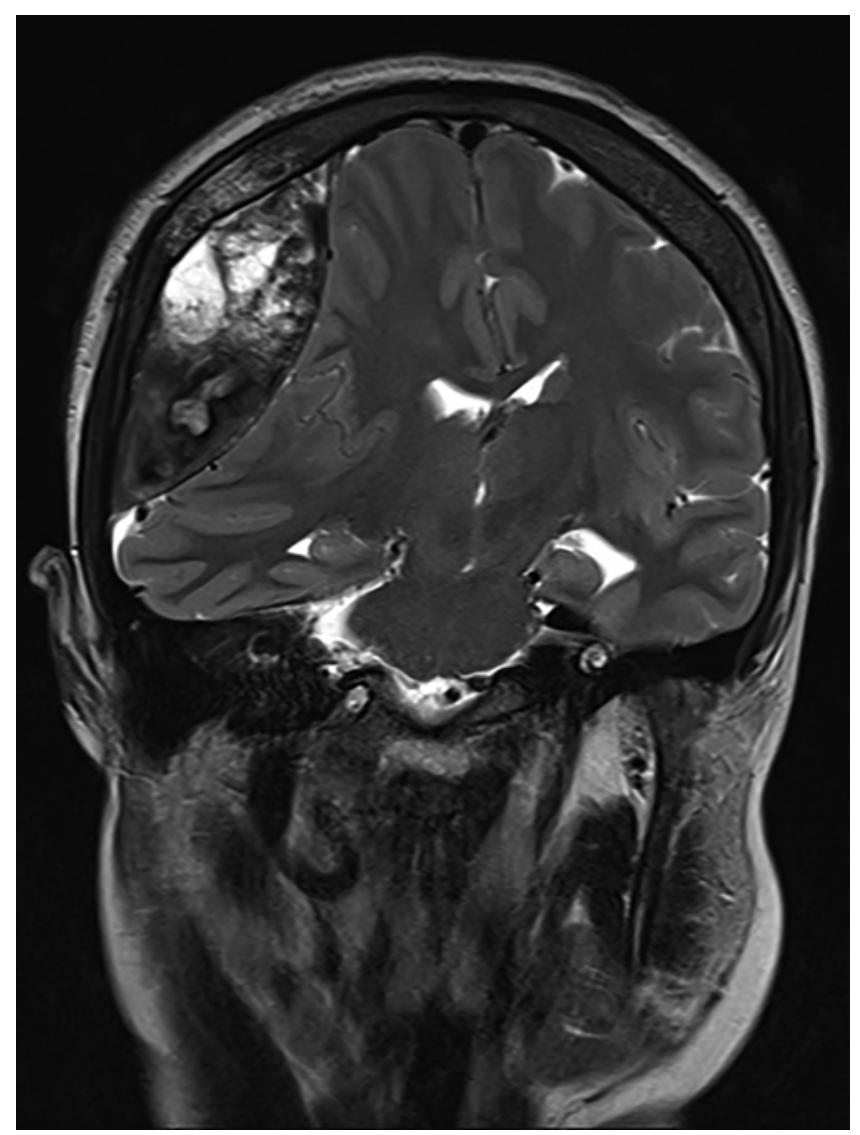

FIG. 1. Case 1. Large right-hemispheric EDH measuring $3.1 \mathrm{~cm}$ in thickness, resulting in right-to-left subfalcine herniation. Blood products are heterogeneous in T2 signal. Abnormal focal signal within the adjacent calvaria is suggestive of adjacent bone infarction.

had also developed profound left hemiparesis, left facial weakness, and a right eye abduction deficit. Given the MRI findings, neurological deficits, and mental status change, she underwent emergency hematoma evacuation, which demonstrated clotted and unclotted blood. The overlying calvaria was not noted to be abnormal in appearance on visual inspection but was not sampled. Specimen pathological investigation revealed multiple fragments of clotted blood. The following day, the patient returned to her neurological baseline and was discharged 7 days after admission. To date, she has not developed a surgical defect or required cranioplasty secondary to bone infarction.

\section{Discussion}

Sickle cell anemia is an autosomal recessive hemoglobinopathy characterized by an abnormality in the oxygencarrying hemoglobin molecule in red blood cells. The red blood cells are predisposed to assume abnormal sickleshape morphology and they obstruct small arteries, causing tissue hypoxia. The clinical consequences include pain crisis, respiratory insufficiency (acute chest syndrome), and strokes. Combined MRI and MRA studies are typically performed for evaluation of headache in patients with sickle cell anemia, specifically if a child presents with vision changes, weakness, or other stroke-like symptoms, or

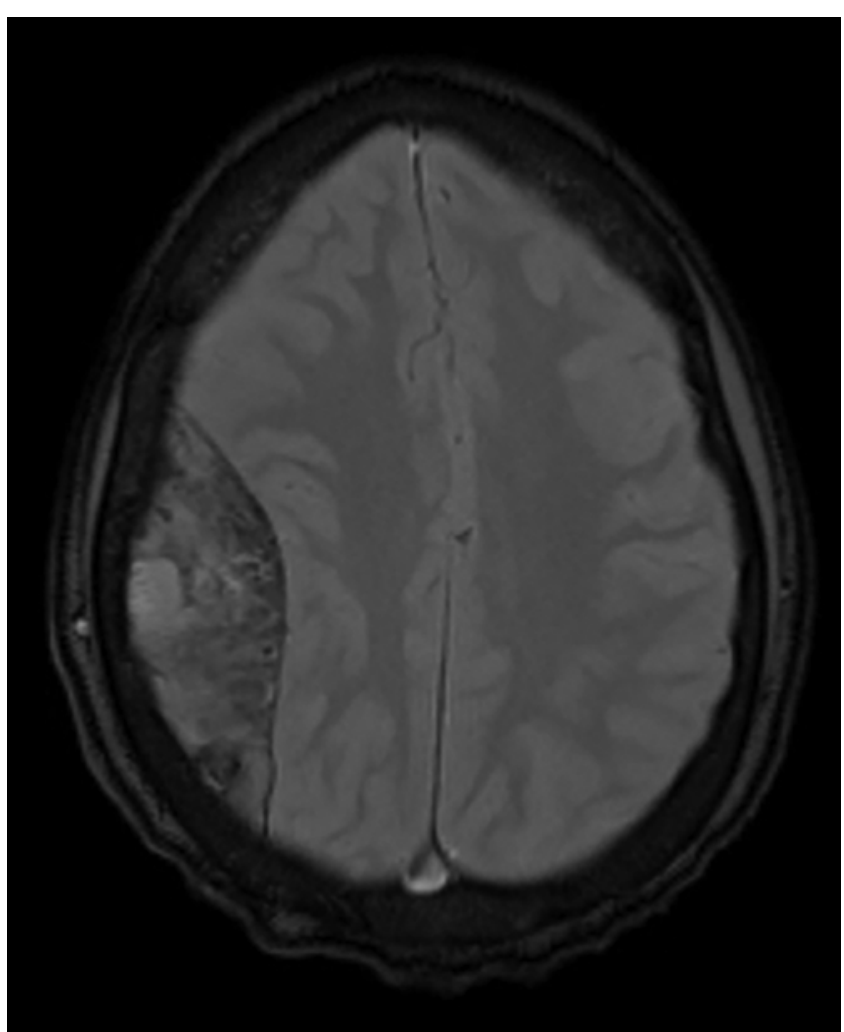

FIG. 2. Case 1. Axial gradient recalled echo imaging demonstrating heterogeneous appearance of blood products.

if the child has had a prior occlusive event. If MRI and MRA are not acutely available, noncontrast CT can be performed. Epidural hematomas are typically thought to be traumatic in origin, often related to laceration of the middle meningeal artery in the setting of fracture.

In this case an adolescent presenting with intractable headache and progressive focal neurological deficits was found to have a large EDH in the absence of a history of trauma. No other reports in patients with sickle cell anemia have described a similar heterogeneous appearance to the $\mathrm{EDH}$, as seen in this case, which often correlates with active hemorrhage. There are a small number of case reports of spontaneous EDHs in the medical literature, approximately 15 cases per review. ${ }^{1-3,5-10}$ The exact incidence is unknown. Even fewer cases are reported with associated calvarial infarction ${ }^{1,6-8,10}$ on CT or MRI as demonstrated in this case. Four case reports reviewed described acute neurological deficits related to EDH due to associated mass effect. ${ }^{3,5,7,9}$ Five of the cases reviewed required emergency decompression, of which approximately half ultimately had favorable outcomes. ${ }^{2,3,5,7,9}$

Furthermore, a second case of spontaneous EDH has been identified (Case 2). A 17-year-old boy presented to the emergency department with an 8-day history of headaches and diffuse scalp edema. He denied a history of trauma or weakness. Evaluation with MRI and MRA without contrast revealed a thickened calvaria thought to be secondary to extramedullary hematopoiesis, with multifocal signal abnormality and patchy restricted diffusion compatible with skull infarction (Fig. 4). Imaging also demonstrated 


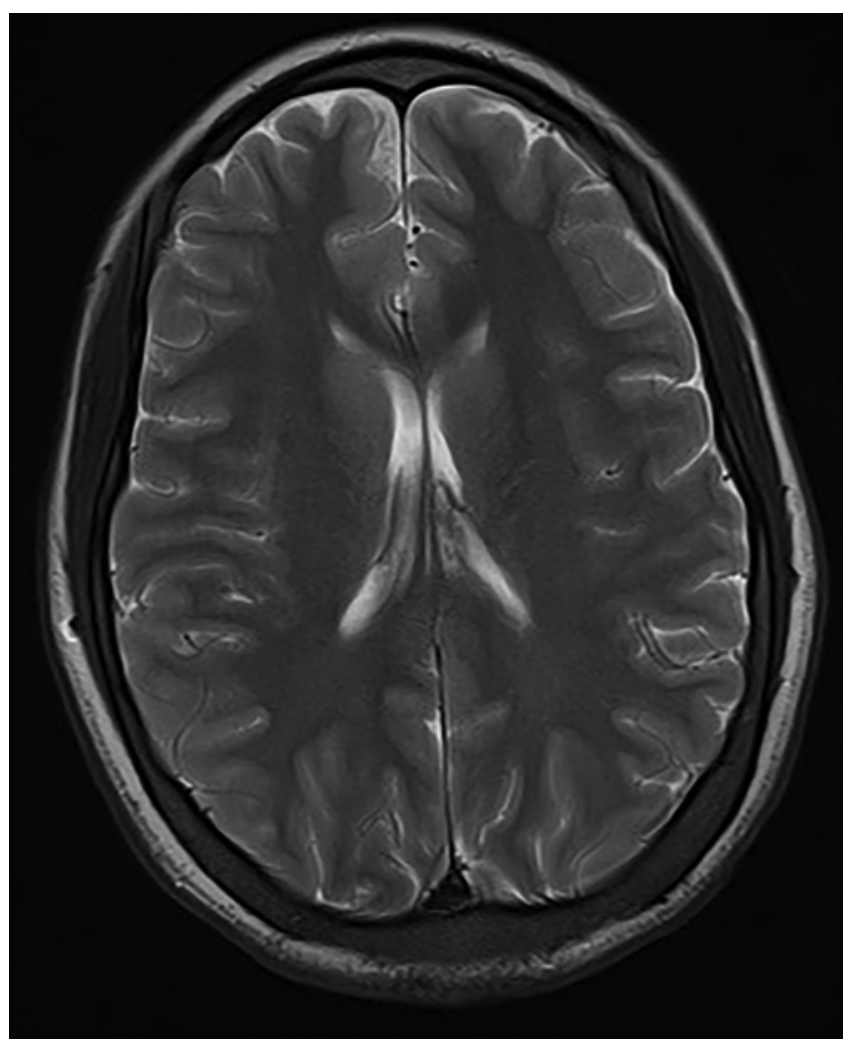

FIG. 3. Case 1. Normal findings on T2-weighted axial MR image of the brain obtained in the same patient 7 months prior to presentation with $\mathrm{EDH}$.

small $\mathrm{T} 1$ and $\mathrm{T} 2$ intense epidural collections along the frontal lobes as well as a large subperiosteal collection compatible with hemorrhage and proteinaceous fluid (Fig. 5). The patient's symptoms were self-limited and resolved without intervention.

Bone infarctions are typically seen in the appendicular skeleton and spine of patients with sickle cell disease. Lateral orbital wall infarctions with subperiosteal collections mimicking abscess are also a recognized complication of

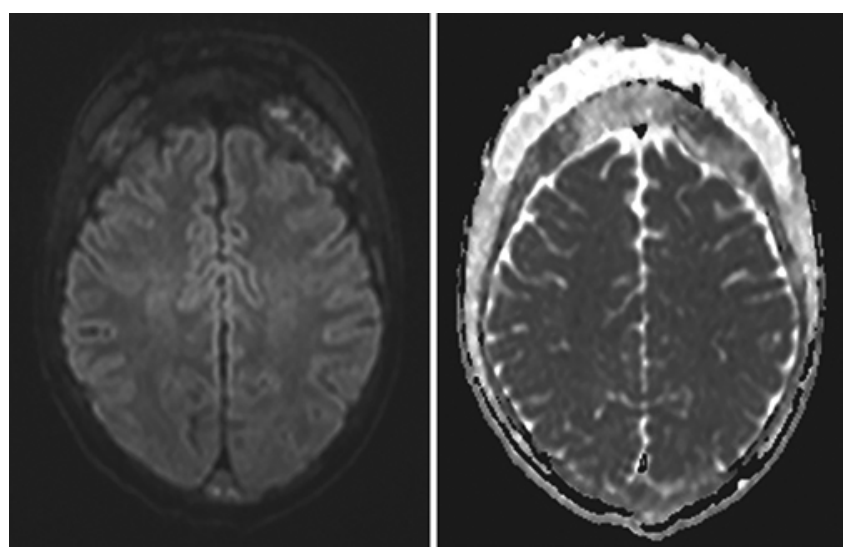

FIG. 4. Case 2. Diffusion-weighted imaging and apparent diffusion coefficient map demonstrating patchy restricted diffusion within the frontal calvaria suggestive of multifocal infarction.

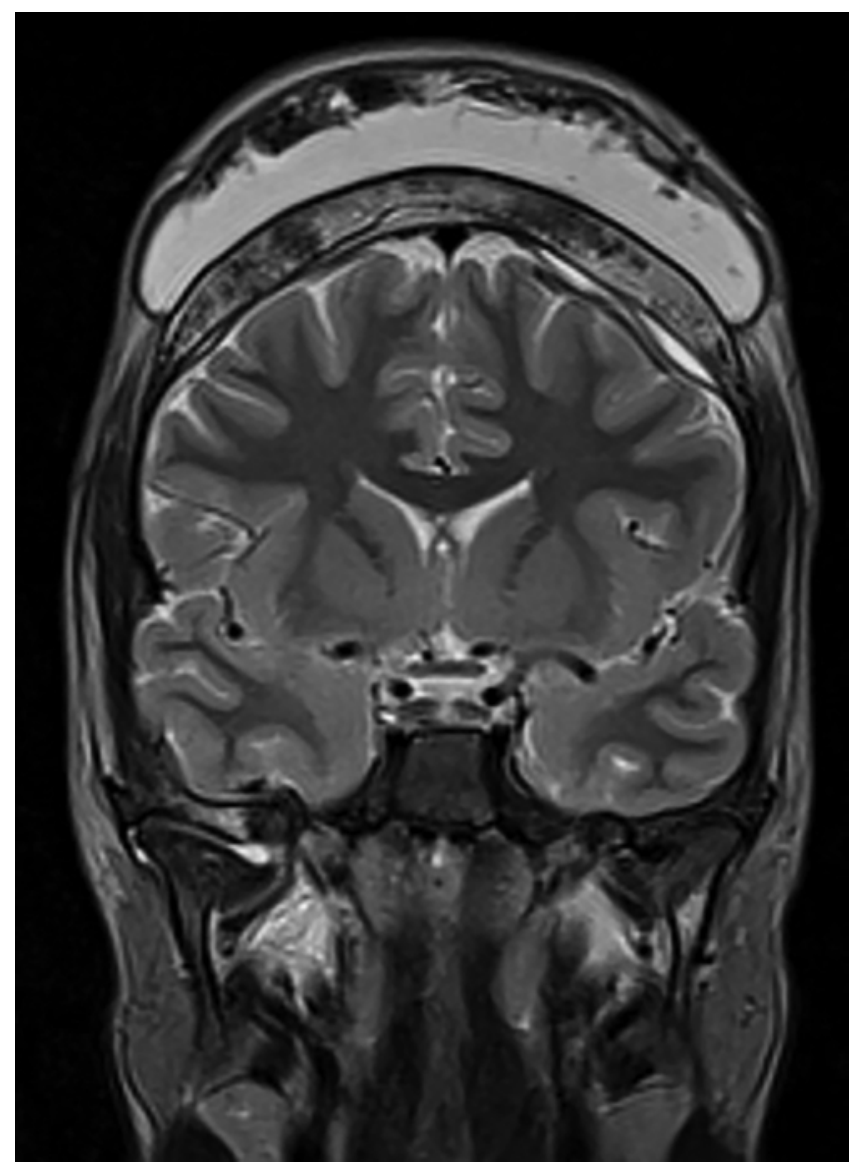

FIG. 5. Case 2. Coronal T2-weighted imaging showing hyperintense epidural collections along the bifrontal lobes as well as a large subgaleal collection of hemorrhage and proteinaceous fluid. Thickening of the calvaria with heterogeneous T2 signal was most compatible with extramedullary hematopoiesis and bone infarction.

sickle cell anemia. Calvarial infarctions are an uncommon complication of sickle cell disease. There are several theories about the mechanism for spontaneous EDHs in this process, including bone ischemia and infarction leading to extracranial or dural periosteal elevation, disruption of the bone margin and vessel wall, and bleeding into the epidural space; ischemia and spontaneous rupture of epidural vessels adjacent to infarcted bone; insufficient venous drainage resulting in venous congestion, rupture of veins, as well as edema and hemorrhage; and abnormal skull anatomy and proliferation of hematopoietic skull tissue in response to anemia resulting in disruption of the skull margins, leading to blood and tissue extravasation into the epidural space. ${ }^{3,5,8,9}$

\section{Conclusions}

This case of a large spontaneous EDH due to calvarial infarction illustrates a rare life-threatening complication of sickle cell anemia in a pediatric patient. This patient had profound focal neurological deficits, which have been rarely reported in this scenario, and responded well to emergency evacuation. Awareness of this rare complication of sickle cell anemia and investigation with appropriate acute 
imaging may ensure prompt diagnosis and treatment. Specifically, this entity should be considered in patients with sickle cell disease not only presenting with neurological deficits but also with severe headaches. On MRI, calvarial marrow heterogeneity may reflect infarction and predispose patients to developing an EDH. As in this case, operative management of EDHs adjacent to marrow infarct can result in a positive outcome and full recovery.

\section{References}

1. Arends S, Coebergh JA, Kerkhoffs JL, van Gils A, Koppen $\mathrm{H}$ : Severe unilateral headache caused by skull bone infarction with epidural haematoma in a patient with sickle cell disease. Cephalalgia 31:1325-1328, 2011

2. Babatola BO, Salman YA, Abiola AM, Okezie KO, Oladele AS: Spontaneous epidural haematoma in sickle cell anaemia: case report and literature review. J Surg Tech Case Rep 4:135-137, 2012

3. Dahdaleh NS, Lindley TE, Kirby PA, Oya H, Howard MA III: A "neurosurgical crisis" of sickle cell disease. J Neurosurg Pediatr 4:532-535, 2009

4. Hines PC, McKnight TP, Seto W, Kwiatkowski JL: Central nervous system events in children with sickle cell disease presenting acutely with headache. J Pediatr 159:472-478, 2011

5. Kalala Okito JP, Van Dammee O, Calliauw L: Are spontaneous epidural haematoma in sickle cell disease a rare complication? A report of two new cases. Acta Neurochir (Wien) 146:407-410, 2004

6. Karacostas D, Artemis N, Papadopoulou M, Christakis J: Case report: epidural and bilateral retroorbital hematomas complicating sickle cell anemia. Am J Med Sci 302:107109, 1991

7. Page C, Gardner K, Height S, Rees DC, Hampton T, Thein SL: Nontraumatic extradural hematoma in sickle cell anemia: a rare neurological complication not to be missed. Am J Hematol 89:225-227, 2014

8. Resar LM, Oliva MM, Casella JF: Skull infarction and epidural hematomas in a patient with sickle cell anemia. J Pediatr Hematol Oncol 18:413-415, 1996

9. Sangle SA, Lohiya RV, Karne SS, Chugh A: Spontaneous epidural hematoma: a rare complication of sickle cell anemia. Neurol India 59:301-302, 2011

10. Tony J, Subramanya G, Kallur KG, Chalapathy AV, Sheshadri S, Lakhkar B: Proptosis, skull infarction and epidural haematoma in sickle thalassemia. Postgrad Med J 71:445, 1995

\section{Disclosures}

The authors report no conflict of interest concerning the materials or methods used in this study or the findings specified in this paper.

\section{Author Contributions}

Conception and design: Soares, Milla. Acquisition of data: Komarla, Milla. Analysis and interpretation of data: Komarla, Soares, Milla. Drafting the article: Komarla. Critically revising the article: Soares, Chern, Milla. Reviewed submitted version of manuscript: all authors. Study supervision: Soares, Milla.

\section{Correspondence}

Rohini Komarla: Emory University School of Medicine, Atlanta, GA.rkomarl@emory.edu. 